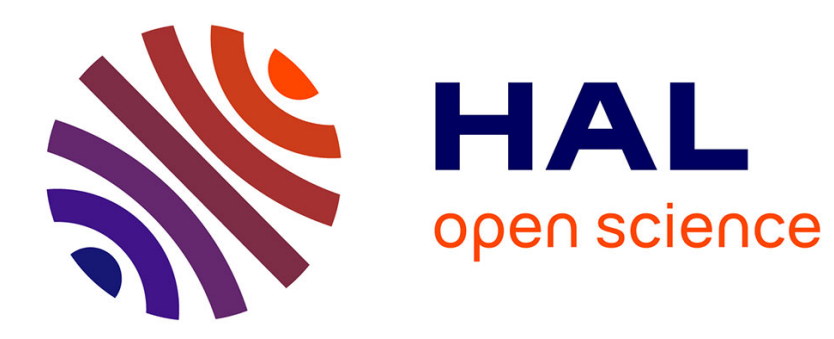

\title{
Développements récents de l'étude du EL2 dans GaAs
}

\author{
H.J. von Bardeleben, B. Pajot
}

\section{To cite this version:}

H.J. von Bardeleben, B. Pajot. Développements récents de l'étude du EL2 dans GaAs. Revue de Physique Appliquée, 1988, 23 (5), pp.725-725. 10.1051/rphysap:01988002305072500 . jpa-00245876

\section{HAL Id: jpa-00245876 https://hal.science/jpa-00245876}

Submitted on 1 Jan 1988

HAL is a multi-disciplinary open access archive for the deposit and dissemination of scientific research documents, whether they are published or not. The documents may come from teaching and research institutions in France or abroad, or from public or private research centers.
L'archive ouverte pluridisciplinaire HAL, est destinée au dépôt et à la diffusion de documents scientifiques de niveau recherche, publiés ou non, émanant des établissements d'enseignement et de recherche français ou étrangers, des laboratoires publics ou privés. 


\section{REVUE DE PHYSIQUE APPLIQUÉE}

\section{Développements récents de l'étude du EL2 dans GaAs}

\section{Introduction}

Le développement de la technologie des circuits intégrés GaAs nécessite entre autres choses un matériau adéquat pour les substrats semi-isolants. Cela est maintenant réalisé par tirage de cristaux semi-isolants de GaAs non dopés par la méthode de Czochralski avec encapsulant liquide. L'activité électrique de ce matériau est dominée par la présence d'un niveau donneur profond associé à un défaut natif, EL2, qui compense les impuretés peu profondes existant dans le cristal. Ceci a été à l'origine de nombreuses études sur ce défaut parues dans les dix dernières années, mais c'est seulement depuis peu que la compréhension de sa structure a fait des progrès significatifs. C'est ce qui nous a incité à rassembler les informations sur EL2 parues dans les deux dernières années dans un numéro spécial de la Revue de Physique Appliquée. Les articles originaux réunis font le point sur la situation à la mi-87. Les thèmes abordés sont : les rapports entre les propriétés de EL2 et les dispositifs (S. Miyazawa et al.), la caractérisation de EL2 en tant que défaut natif et sa distribution dans le cristal (Y. Mochizuki et al. ; M. Bonnet et al. ; J. P. Fillard), les propriétés optiques et la métastabilité induite optiquement (M. Kaminska ; B. Dischler et al.), la modélisation de la structure microscopique de EL2 (D. Stievenard et al. ; B. K. Meyer ; G. A. Baraff) les réactions en phase solide où intervient EL2 (G. Guillot ; S. Makram-Ebeid et al.) et pour finir, un point de vue critique sur les différents modèles proposés pour EḶ2 (J. C. Bourgoin et al.).

Les éditeurs de ce numéro spécial remercient vivement les auteurs qui y ont contribué et espèrent que cette mise à jour stimulera la recherche sur ce défaut important.

H. J. von Bardeleben, B. Pajot 